\title{
Esclerosis múltiple: análisis de necesidades y calidad de vida de los afectados y su entorno
}

\author{
Multiple Sclerosis: Needs analysis and \\ quality of life of affected people and their \\ environment
}

\section{Resumen}

La evolución en el paradigma desde el que se contempla la discapacidad, y en concreto la esclerosis múltiple, ha estado marcada por los cambios en el concepto de salud, en el avance en los derechos sociales subjetivos y en la implantación progresiva de un modelo inclusivo para su abordaje. Con esta investigación se pretende responder a las preguntas de ese nuevo paradigma, revisando la situación epidemiológica, los estudios anteriores, y el desarrollo de una metodología complementaria desde lo cuantitativo y lo cualitativo. El trabajo de campo -desarrollado en el ámbito de la Comunidad Foral de Navarra- y su posterior análisis nos ofrece una prevalencia considerablemente mayor que la habitualmente manejada hasta ahora, un perfil con características bastante estables en el que destaca el importante grado incapacitante y su correlato socioeconómico, y el aumento en los tratamientos farmacológicos respecto a datos anteriores. La ausencia de una respuesta curativa y el avance en las terapias paliativas exigen, como paso ineludible, la necesidad de poner el foco en aquellos aspectos que mejoren la calidad de vida de las personas afectadas y su entorno.

\section{Palabras clave}

Esclerosis múltiple, discapacidad, síntomas asociados, personas afectadas, análisis de necesidades, calidad de vida, cuidados sociosanitarios, empoderamiento.

\section{Abstract}

The evolution of the disability paradigm, and its conceptualization of multiple sclerosis, has been marked by changes in the health concept, in the development of subjective social rights and the progressive implementation of an inclusive model for its approach.

This research tries to answer questions about this new paradigm, reviewing the epidemiological situation, previous studies, and developing a complementary quantitative and qualitative methodology. Fieldwork-developed at the Autonomous Community of Navarre- and subsequent analysis offer a significantly higher prevalence than usually handled so far, a fairly stable profile characterized by a severe degree of disablement with strong socioeconomic correlates and a proliferation of pharmacological treatments in comparison to earlier data. The lack of a healing response and the progress in palliative therapies require, as an unavoidable step, the need to focus on those aspects that improve the quality of life of affected people and their environment.

\section{Key words}

Multiple sclerosis, disability, associated symptoms, affected people, needs analysis, quality of life, social and medical cares, empowerment.

\section{José Carrón \\ <jose@ecarron.net>}

Departamento de Psicología y Sociología, Universidad de Zaragoza

\section{Javier Arza}

<javier.arza@unavarra.es>

Departamento de Trabajo Social, Universidad Pública de Navarra

Para citar:

Carrón, J. y Arza, J. (20I3):

"Esclerosis múltiple: análisis de necesidades y calidad de vida de los afectados y su entorno". Revista Española de Discapacidad, I (2): 59-75.

<http://dx.doi.org/IO.5569/23405 IO4.01.02.04>

Fecha de recepción: 10-09-20I3 Fecha de aceptación: O4-I2-2OI3 


\section{Introducción}

\subsection{La esclerosis múltiple: concepto e impacto}

La esclerosis múltiple puede describirse, desde una perspectiva divulgativa, como un trastorno crónico del sistema nervioso central -formado por el encéfalo y la médula espinal- caracterizado por la pérdida progresiva de mielina. Ésta es una sustancia blanca que actúa como una vaina recubriendo los nervios. Desde el momento que va desapareciendo comienzan a producirse placas de tejido endurecido, proceso conocido como esclerosis. En consecuencia, los impulsos nerviosos se interrumpen periódicamente llegando a hacerlo en ocasiones de forma definitiva (Compston y Coles, 2008; Correale et al., 2002).

Suele aparecer entre los 20 y 40 años (Simone et al., 2000), con una preponderancia de mujeres -en torno a $2 / 3$ de los casos (Duquette et al., I992)- y es una enfermedad con una gran variabilidad en cuanto a los síntomas (Ede, 2003; Ademna, 2006; Felem, 2007; Fedema, 2008), dependiendo de las áreas del sistema nervioso central que estén afectadas. Algunas manifestaciones clínicas frecuentes que se presentan en forma de crisis o brotes incluyen, según las ocasiones y las personas afectadas, parálisis, ataxia, temblores, debilidad, hormigueo, problemas visuales, pérdida de fuerza o sensibilidad, problemas en la función sexual, problemas de memoria a corto plazo, alteraciones urológicas,... (Raus, I998; Delgado-Mendilívar et al., 2005).

La esclerosis múltiple es, habitualmente, una enfermedad con un largo recorrido. Su aparición en las primeras etapas de la edad adulta, y su carácter no letal, hace que las personas que la padecen tengan por delante una larga esperanza de vida, durante la cual tendrán que convivir con la enfermedad y precisarán apoyo y cuidados por parte de su entorno, del sistema sociosanitario y de la sociedad en general.

A día de hoy no se dispone de ninguna respuesta curativa para la esclerosis múltiple, pero es cierto que se ha avanzado de manera notable en el tratamiento clínico y rehabilitador y en la mejora de los síntomas, logrando incluso detener la evolución con intervenciones precoces mediante los tratamientos farmacológicos disponibles, aunque las secuelas no son reversibles (Banwell et al., 2013; Giovannoni, 2013).

De todo ello no es difícil inferir, por un lado, el gran impacto emocional que tiene lugar a partir del diagnóstico y, por otro, la significativa repercusión en el proyecto vital de las personas afectadas y sus familias: coincide con la etapa más productiva en la vida de una persona, supone una fuerte inversión de recursos -económicos, de tiempo, de esfuerzos...- y en el caso de las mujeres, sobrerrepresentadas en esta enfermedad, implica una discriminación añadida a otras relacionadas con el género.

\subsection{La esclerosis múltiple: epidemiología}

Históricamente se ha dispuesto en España de numerosos estudios locales que, con diferentes metodologías, han ofrecido resultados significativamente divergentes entre sí sobre la prevalencia de la esclerosis múltiple en nuestro país (Mallada, I999). A nivel estatal, la información epidemiológica que encontramos en diversas publicaciones del sector se mueve generalmente en estimaciones globales que se repiten de una a otra, sin establecer claramente el origen de la misma. Así, por ejemplo, en la introducción de una encuesta estatal realizada en 2005-06 (Felem, 2007) se cita textualmente que en ese momento “en España, la prevalencia media se puede cifrar en 40-50 casos por cada I00.000 habitantes..." aunque sin especificar el posible origen del dato. También en la página web de la Sociedad Española de Neurología se refleja una información similar “... de acuerdo con los datos manejados por la S.E.N., en España unas 46.000 personas padecen esclerosis múltiple..." (Sociedad Española de Neurología, 20I3). Sin embargo, si se acude directamente a fuentes estadísticas en determinados casos de origen secundario, como los registros del Departamento de Salud del Gobierno de Navarra (Gobierno de Navarra, 2010), o en otros de origen primario, como la Encuesta sobre Discapacidad, Autonomía 
personal y situaciones de Dependencia (EDAD) del Instituto Nacional de Estadística del año 2008 (INE, 2008), los datos disponibles permiten establecer unos niveles de impacto sensiblemente más elevados que los manejados hasta el momento.

Para Navarra, ámbito específico de la presente investigación, se dispone de información con metodología censal provista por el Departamento de Sanidad del Gobierno Foral (Gobierno de Navarra, 20Io) sobre los casos que figuran en sus registros. Para 20Io son un total de 497 personas diagnosticadas de esclerosis múltiple. Si se toma en cuenta la población de derecho para ese año en Navarra (636.924 habitantes), se obtiene una tasa de 78 casos por I00.000 habitantes que situaría esta prevalencia en una zona de riesgo alto según la clasificación internacional establecida por Kurtzke (1993; I997). En cuanto a la distribución por sexo, la sobrerrepresentación femenina que citábamos también se refleja en los datos navarros, donde la prevalencia entre ellas es de I2 I casos por cada I00.000 mujeres mientras que los varones están afectados 40 de cada I00.000 navarros.

Tabla 1. Personas afectadas de esclerosis múltiple en 2010 en Navarra

\begin{tabular}{|l|c|c|c|}
\hline Edad & Varones & Mujeres & $\begin{array}{c}\text { Ambos } \\
\text { sexos }\end{array}$ \\
\hline 0 a 14 & 0 & 1 & $\mathbf{1}$ \\
\hline 15 a 44 & 48 & 157 & $\mathbf{2 0 5}$ \\
\hline 45 a 64 & 60 & 171 & $\mathbf{2 3 1}$ \\
\hline 64 y + & 15 & 45 & 60 \\
\hline Total & $\mathbf{1 2 3}$ & $\mathbf{3 7 4}$ & $\mathbf{4 9 7}$ \\
\hline Casos/100.000 & $\mathbf{4 0}$ & $\mathbf{1 2 1}$ & $\mathbf{7 8}$ \\
\hline
\end{tabular}

Fuente: Elaboración propia a partir de los datos del Departamento de Salud. Gobierno de Navarra.

La distribución por edades responde asimismo a los patrones más conocidos para esta enfermedad (Ede, 2003; Ademna, 2006; Felem, 2007; Fedema, 2008). La mayor parte de los casos para 2010 en Navarra (Tabla I) se sitúan entre los 45 y 64 años (un 46,5\%), seguida de la franja de I 5 a 44 años (el 4I,2 \%). Un I 2,I \% tiene 64 ó más años y solamente uno de los casos no había aún cumplido los I 5 años.

Para la comparación de los datos navarros con el nivel estatal nos hemos encontrado con serias dificultades ante la ausencia de datos globales provenientes de registros primarios. La aproximación más fiable de la que se dispone es la Encuesta EDAD 2008 del Instituto Nacional de Estadística (INE, 2008), en la que se entrevistó a más de 270.000 personas de 6 ó más años. Asumiendo que dicha Encuesta refleja autodeclaraciones y no diagnósticos clínicos, resulta sorprendente que la estimación que realiza para el ámbito navarro, aún siendo un relativamente bajo volumen de población y por tanto un mayor error de muestreo, es muy similar a los datos que se habían obtenido de fuentes primarias. Cifra los afectados por esclerosis múltiple en 78 casos por ı००.000 habitantes, lo que representaría un total de 453 personas afectadas. Haciendo extensiva esa fiabilidad a los datos estatales (con mayor fuerza estadística al disminuir el error muestral por el tamaño considerablemente más elevado) se encuentran para la esclerosis múltiple I 44 casos por I 00.000 habitantes. La proyección en el conjunto de la población (Tabla 2) elevaría a más de 60.000 personas de 6 ó más años afectadas por la enfermedad a nivel estatal, con una estimación estadística que, si acaso, podría estar sesgada a la baja en tanto en cuanto las personas afectadas con discapacidad leve o nula podrían no estar incluidas en estos resultados.

Tabla 2. Estimación de afectados por esclerosis múltiple en 2008 (6 y + años)

\begin{tabular}{|l|c|c|c|}
\hline & Varones & Mujeres & Ambos sexos \\
\hline $\begin{array}{l}\text { España. } \\
\text { Casos/100.000 }\end{array}$ & 83 & 204 & $\mathbf{1 4 4}$ \\
\hline $\begin{array}{l}\text { Navarra. } \\
\text { Casos/100.000 }\end{array}$ & 68 & 87 & $\mathbf{7 8}$ \\
\hline $\begin{array}{l}\text { España. Total } \\
\text { afectados }\end{array}$ & 17.300 & 43.600 & $\mathbf{6 0 . 9 0 0}$ \\
\hline $\begin{array}{l}\text { Navarra. Total } \\
\text { afectados }\end{array}$ & 199 & 254 & $\mathbf{4 5 3}$ \\
\hline
\end{tabular}

Fuente: Elaboración propia a partir de los datos de la encuesta EDAD 2008. INE. 
En los datos estatales de EDAD 2008 puede constatarse una vez más la contundente sobrerrepresentación de mujeres afectadas en una relación de siete de cada diez casos ( 204 frente a 83 por I00.000 entre los varones). Para el caso específico de la población navarra, al segmentar por sexos, el notable aumento del error muestral en una población estadísticamente insuficiente impide valorar la falta de ajuste con los datos primarios ya comentados.

\subsection{Cambio de paradigma en el ámbito de la discapacidad}

El análisis de las consecuencias de patologías con un alto potencial discapacitante, como es el caso de la esclerosis múltiple, no puede llevarse a cabo sin hacer referencia al cambio de paradigma que está teniendo lugar en las últimas décadas en el ámbito de la discapacidad en su conjunto, y que se articula en torno a tres constructos altamente significativos:

- El concepto de salud, en el que está en marcha un cambio de un modelo sanitarista a un modelo bio-psico-social que contempla aspectos como la prevención, el apoyo emocional, la salud pública o las modificaciones ambientales (OMS, 2009; Pineault et al., 2003; OMS, I978; Ponte, 2008).

- Los servicios sociales y, en general, los derechos sociales a través de un avance progresivo en la consideración de la atención a las necesidades sociales de la ciudadanía como derechos subjetivos y de los servicios sociales como un sistema universal, garantista y de calidad (España, 2006; Navarra, 2006).

- La concepción y el abordaje de la discapacidad, que asiste a un cambio de enfoque desde un modelo individualista/ asistencial/rehabilitador, a un modelo inclusivo que busca, además del trabajo individual, incidir sobre las creencias sociales y eliminar las barreras que impiden la participación social plena a las personas con discapacidad (Pérez, 2006;
Gobierno de España, 20I I; Huete y

Díaz, 2009).

Es necesario poner de manifiesto que, en todo caso, el proceso de transformación iniciado a través de este cambio de paradigma no ha sido en el pasado lineal, enfrentándose a numerosas dificultades, resistencias, contradicciones,... En estos momentos, la puesta en cuestión de aspectos nucleares del modelo sociosanitario, permite incluso hablar de retrocesos significativos en su desarrollo.

En el ámbito de la esclerosis múltiple, la plasmación de este cambio de paradigma se ha reflejado de manera contundente en los Principios Internacionales para promover la calidad de vida de las personas con esclerosis múltiple (MSIF, 2002). Tomando como referencia el concepto de calidad de vida de la OMS “percepción que tiene un individuo de su situación en la vida, dentro del contexto cultural y de valores en que vive y en relación con sus objetivos, expectativas, valores e intereses" (WHOQOL, I995), los parámetros médicos y asistenciales pasan a constituir un elemento más dentro de un planteamiento estratégico dirigido a lograr una mejora significativa en la calidad de vida de los afectados. Se estructuran en torno a Io principios: independencia y empoderamiento; asistencia médica; asistencia continua (cuidados de larga duración); promoción de la salud y prevención de la enfermedad; apoyo a miembros de la familia; transporte; empleo y actividades de voluntariado; pensiones y ayudas económicas por discapacidad; educación; vivienda y accesibilidad a edificios comunitarios cuya sola enumeración nos pone en la pista de este cambio de enfoque.

Es necesario que este cambio de paradigma vaya impregnado los intersticios de las diversas estrategias de investigación, como así ha ocurrido en el estudio que sirve como base al presente artículo. La investigación no debe limitarse a analizar la incidencia de la discapacidad en las personas afectadas, sino también en su contexto; debe abordar las necesidades globales de la persona y su entorno, no limitándose a los aspectos médicos 
y asistenciales; debe servir para detectar las necesidades que el Estado tendrá que garantizar como derechos sociales; etc.

\subsection{La investigación sobre las características, necesidades y problemáticas de los afectados}

Al margen de algunas investigaciones ya citadas, centradas específicamente en datos epidemiológicos, en esta última década se han llevado a cabo varios estudios con el objetivo de profundizar en las características, necesidades y problemáticas de los afectados por esclerosis múltiple y en su entorno familiar y social.

En el estudio llevado a cabo en 2003 para la Fundación Eugenia Epalza (Ede, 2003) en el País Vasco ( 242 entrevistados), el $74 \%$ eran mujeres, con un $63 \%$ de los encuestados entre los $30 \mathrm{y}$ 49 años y el $70 \%$ tenía entre 20 y 40 años en el momento del diagnóstico. Los síntomas más frecuentes eran los trastornos motores (un $58 \%$ ) seguidos de la falta de equilibrio y la fatiga. El $79 \%$ poseía certificado de discapacidad, y un $46 \%$ en un grado superior al $60 \%$. El $47 \%$ había utilizado alguna terapia alternativa -herboristería un $44 \%$, homeopatía un $36 \%$, o acupuntura un $34 \%-$. El $44 \%$ utilizaba productos de apoyo -un $62 \%$ silla manual/ silla eléctrica, un $34 \%$ muleta-. El $60 \%$ recibía ayuda de terceras personas, fundamentalmente de su pareja; el $7 \%$ disponía de un cuidador externo. El $47 \%$ necesitaba ayuda para las actividades de la vida diaria, destacando especialmente el ir de compras, barrer o realizar gestiones fuera de casa. El $34 \%$ se encontraba trabajando, un $37 \%$ disponía de una pensión y un $6 \%$ se encontraba desempleado; el I $2 \%$ se dedicaba a las tareas del hogar. Un 45 \% había dejado de trabajar a raíz de diagnosticarle la enfermedad. El 3 I \% tenía adaptado el acceso a su vivienda y un $46 \%$ no lo tenía aunque lo necesitaba. En cuanto a la vivienda en sí el I7\% si la tenía adaptada y un 3I \% la necesitaba. El $6 \%$ tenía adaptado su vehículo, mientras que un $20 \%$ no lo tenía aunque lo necesitaba. Un I3 \% utilizó apoyo psicológico cuando le diagnosticaron la enfermedad, otro I $7 \%$ lo había hecho de manera puntual desde entonces, y otro $6 \%$ de manera continuada hasta ahora. Los servicios más demandados eran, por este orden, fisioterapia, asistencial social y ayuda a domicilio.

En un estudio sobre la calidad de vida de las personas afectadas por esclerosis múltiple, Delgado-Mendilívar y colaboradores (DelgadoMendilívar et al., 2005) trataron de observar cuáles eran las áreas más afectadas por la enfermedad y en qué medida el tratamiento podía mejorar su calidad de vida. De acuerdo con sus resultados, habiendo dividido a las personas afectadas en tres grupos según su puntuación en las escala EDSS o de Kurtzke (I993), el dolor corporal y la vitalidad son las dimensiones más afectadas en un estadio temprano de la enfermedad. Los ítems relacionados con la salud física serían los más influidos por la evolución de la enfermedad y no ocurriría tanto en aspectos relacionados con la salud mental salvo en lo que afecta a la función emocional. Por último, dentro del área de salud física -función física, rol físico, dolor corporal, salud general- y del área de salud mental -vitalidad, función social, rol emocional, salud mental- la aplicación de un tratamiento rehabilitador se mostró especialmente efectivo en relación con las dimensiones de rol físico y de rol emocional.

En 2005-2006 la Federación Española para la Lucha contra la Esclerosis Múltiple (FELEM) y el Ministerio de Trabajo y Asuntos Sociales impulsaron la realización de una encuesta a nivel estatal cuya población diana eran las personas asociadas en la Federación. Para llevarla a cabo "se eligió una muestra de 500 personas (...) 375 personas socias de las asociaciones que componen FELEM y I 25 personas no asociadas" (Felem, 2007: I7). Además se realizaron entrevistas, por cada asociación, a uno o dos neurólogos de referencia y a un responsable de la misma. El perfil de afectado puede trazarse con los siguientes datos: el 68,4\% eran mujeres; siete de cada diez (un 71,०\%) tenía entre 30 y 54 años; la mitad (un 46,8\%) llevaba diagnosticado entre 7 y I 6 años y en otro 3 I, $0 \%$ de los casos el diagnóstico era menor de 7 años; en más del $50 \%$ de los casos la enfermedad cursaba 
de manera remitente-recurrente; un 48,6\% precisaban apoyos para deambular y un $23,2 \%$ silla de ruedas para desplazarse; ocho de cada diez (el 78,6\%) tenía certificado de discapacidad y un $37,8 \%$ de los entrevistados con grado de $65 \%$ o superior. El $26,5 \%$ estaban trabajando, siendo un $46,2 \%$ pensionistas y otro 8 , I $\%$ se encontraban en paro. Además del servicio de neurología, utilizado prácticamente por todos los afectados, el más demandado era fisioterapia (un 62,4\%). Coincidiendo con lo expresado por los neurólogos, los problemas físicos más frecuentes eran la fatiga, la pérdida de fuerza, las disfunciones sexuales y la incontinencia urinaria; entre los emocionales: la depresión seguida de la ansiedad; en cuanto al deterioro cognitivo, citaban las dificultades para concentrarse, los trastornos de memoria, la capacidad de planificación y el lenguaje no fluido. Un 57,3\% tenía realizadas adaptaciones en el hogar y un $34,0 \%$ necesitaba adaptar la vivienda a sus necesidades.

Ampliando el foco a nivel continental, el Código Europeo de Buenas Prácticas (EMSP, 2008) resalta entre los elementos más significativos a nivel europeo en relación con la esclerosis múltiple: la baja tasa de personas afectadas en Europa con acceso a medicación efectiva contra la enfermedad (un $28 \%$ ); la escasez de médicos especializados (en Irlanda solamente hay I4 neurólogos especialistas); o los índices de ocupación de los afectados, 2 ó 3 veces más bajos que el resto de la población.

Para finalizar el repaso de estos antecedentes, en el año 2006, la asociación de esclerosis múltiple de Navarra (ADEMNA) llevó a cabo un estudio (Ademna, 2006) dirigido a los asociados diagnosticados en el que se reflejaba la situación de las personas afectadas -y de su entorno- en los siguientes términos: tres cuartas partes eran mujeres(un 73,8\%), básicamente entre los 40 y 59 años (el 58,9\%), diagnosticados mayoritariamente entre los $25 \mathrm{y}$ 40 años (un $52,2 \%$ ), con un 76,6\% siguiendo un tratamiento farmacológico y un $36,4 \%$ que había utilizado en alguna ocasión tratamientos no convencionales, especialmente herboristería y acupuntura. El 59,8\% tenía reconocida una discapacidad (de ellos el 33,7\% del 65\% o más). El 40,2 \% utilizaba algún tipo de producto de apoyo, especialmente muletas y sillas manuales. Las ayudas personales que recibían provenían fundamentalmente (en un $45,8 \%$ ) de su pareja, disponiendo de cuidadores externos en un $7,5 \%$ de los casos.

Un 44,2 \% necesitaba ayuda para las actividades de la vida diaria. Especialmente para ir de compras (el 77,5\% de los casos), para planchar (un $69,4 \%$ ) o para levantarse o acostarse (un $63,3 \%)$.

El 24,3\% estaba trabajando, un 28,2 \% con pensión, y un I $4,2 \%$ se encontraba en paro. El Iо, $8 \%$ se dedicaba a las tareas del hogar. Para el $54,2 \%$ la enfermedad había supuesto tener que dejar el trabajo. Un 19,6\%, el conocimiento de la enfermedad le generó problemas en el entorno laboral.

El 54,9\% tenía el acceso a la vivienda adaptado a sus necesidades y el $36,5 \%$ necesitaba adaptarlo pero no lo tenía hecho. Solamente el I 4, 8 \% tenía adaptado el vehículo y un $34,7 \%$ no lo tenía aunque lo necesitaba.

El 3,9\% utilizó apoyo psicológico solamente cuando le diagnosticaron la enfermedad, un I 3,7\% lo había utilizado desde entonces de manera puntual y otro $5,9 \%$ de manera continua desde entonces.

Los servicios más demandados eran fisioterapia (el 67,0\%), asistencia social (un 33,6\%), yoga (30,I \%), talleres ocupacionales $(26,6 \%)$ y atención psicológica (2I,5\%).

Seis años después de ese primer estudio, ADEMNA decidió realizar una nueva investigación con el objetivo de analizar la situación y los avances -o retrocesos- llevados a cabo durante ese periodo de tiempo, así como poder reforzar, reorientar y proponer nuevas acciones de mejora de la calidad de vida para las personas afectadas por esta enfermedad.

Por ello, después de revisar algunos de los estudios más relevantes sobre la situación 
sociosanitaria de los afectados por esclerosis múltiple y su entorno, en el siguiente apartado se presentan la metodología y los principales resultados de la investigación llevada a cabo en 2012 sobre las personas que se encontraban diagnosticadas por esta enfermedad en Navarra.

\section{Material y método}

La metodología del estudio global ha sido mixta, combinando tanto técnicas cuantitativas como cualitativas. El punto de partida se fundamentó en un amplio análisis documental de fuentes secundarias y una profunda investigación sobre la percepción y la dinámica social del concepto de discapacidad en su conjunto y su plasmación detallada en el entorno de la esclerosis múltiple. A continuación se llevó a cabo una encuesta, cuya metodología se detalla posteriormente, a personas diagnosticadas en la Comunidad Foral de Navarra, con un total de I 40 respuestas válidas. El cuestionario estaba centrado en revisar los principales datos sociodemográficos y médico-sanitarios de los entrevistados, así como trazar un perfil de su situación en relación con los ítems incluidos en los Principios Internacionales para promover la calidad de vida de las personas con esclerosis múltiple (MSIF, 2002). En la siguiente fase se realizaron 20 entrevistas semiestructuradas $-\mathrm{I} 5$ a personas afectadas y 5 a profesionales sanitarios-y 6 grupos focales -profesionales del sistema sociosanitario, profesionales de ADEMNA, parejas de personas afectadas y padres o madres de personas afectadas-, finalizando el estudio con un análisis DAFO y la elaboración de una serie de recomendaciones y propuestas de avance.

En resumen, el proceso metodológico ha contado con los siguientes pasos:

- Análisis documental de fuentes secundarias.

- Análisis cuantitativo. Encuesta a todas las personas con esclerosis múltiple socias de ADEMNA. I 40 cuestionarios válidos.
- Análisis cualitativo. 20 entrevistas semiestructuradas ( 5 a personas afectadas y 5 a profesionales sanitarios) y 6 grupos focales (profesionales del sistema sociosanitario, parejas de personas afectadas y padres o madres de personas afectadas).

- Análisis DAFO, recomendaciones y propuestas de avance.

La encuesta propiamente dicha, cuyo análisis constituye el armazón central del presente artículo, estaba dirigida a personas afectadas y diagnosticadas por esclerosis múltiple en el ámbito de la Comunidad Foral de Navarra. Por cuestiones de confidencialidad respecto a aspectos privados de salud y, por ende, de posibilidad de localización, el universo poblacional se ha circunscrito a las personas socias de ADEMNA diagnosticadas con la enfermedad. Esto ha supuesto un total de 207 casos a los que nos hemos dirigido con una estrategia censal, con lo que no ha sido necesario hacer un diseño muestral representativo. El primer contacto ha sido realizado por ADEMNA para preguntarles por su disposición a participar en el estudio y, en caso afirmativo, si les parecía adecuada una entrevista personal o preferían que les fuera enviado el cuestionario por correo y autorrellenarlo de manera privada.

El resultado final ha sido de $\mathrm{I} 4 \mathrm{O}$ cuestionarios completados (un 67,6\% del universo fijado). Los casos no conseguidos lo han sido por las siguientes razones: en un $16,9 \%$ el cuestionario postal no fue devuelto; en un $9,7 \%$ no se pudo localizar al afectado; un 2,9\% rechazó participar en el estudio; y otro 2,9\% estaban en un estado de salud que no permitía su participación. El $65,0 \%$ de los cuestionarios cumplimentados lo fueron mediante entrevista personal y el $35,0 \%$ restante fueron rellenados por la persona encuestada y devueltos por correo.

En cuanto al contenido del cuestionario, constaba de 90 preguntas organizadas en ocho apartados: datos generales, en el que se incluían preguntas de tipo sociodemográfico, pero también otras referidas a la enfermedad (año de diagnóstico, síntomas, tipo de tratamientos, etc.); ayudas y prestaciones económicas por dependencia; 
independencia y empoderamiento, con preguntas muy orientadas a detectar necesidades de ayuda personales en las actividades de la vida diaria; empleo, relaciones sociales y actividades de ocio y voluntariado; vivienda y accesibilidad; promoción de la salud, calidad de vida y prevención de la enfermedad; asistencia médica y continua; y valoración de ADEMNA.

El trabajo de campo fue realizado entre el o5 de septiembre y el 30 de noviembre de 20 I I por un equipo de cinco profesionales supervisados por el equipo investigador. La explotación de los datos ha sido llevada a cabo con el paquete estadístico SPSS I 5.0 .

\section{Resultados}

\subsection{Perfil sociodemografico}

El perfil sociodemográfico que se refleja a partir de la encuesta ofrece los siguientes resultados: la edad media de los afectados por esclerosis múltiple es de 46,5 años, con un rango que oscila entre los 25 y 80 años pero en el que seis de cada diez están ubicados en la horquilla de 35 a 54 años (Figura I). Por sexo, siete de cada diez afectados son mujeres (el 70,7\%).
Viven básicamente en pareja y solamente un $8,0 \%$ lo hacen solos, frente al porcentaje del I $8,8 \%$ entre la población general española (INE, 20I3). El 64,3\% han tenido hijos, destacando que cuatro de cada diez (un $38,9 \%$ ) los ha tenido cuando la enfermedad ya había sido diagnosticada.

Solamente un $4,3 \%$ no había conseguido terminar los estudios primarios, un $32, \mathrm{I} \%$ sale del sistema educativo con estudios primarios, un $32, \mathrm{I} \%$ con educación secundaria finalizada, y el 3 I, $4 \%$ ha obtenido alguna titulación universitaria.

En cuanto a su situación laboral, tres de cada diez (un $30,7 \%$ ) trabajan fuera del hogar, uno de cada diez (el I I,4\%) se encontraba en desempleo, la tercera parte (el 33,6\%) recibía una pensión de invalidez/incapacidad permanente, el I 2,8 \% recibía una pensión de jubilación y un $7,9 \%$ se dedicaba a las tareas del hogar. La media de ingresos mensuales por unidad familiar es de 2.040 euros, pero la distribución es notablemente heterogénea: tres de cada diez hogares no superan los I.200 euros de ingresos; aproximadamente la cuarta parte (el 23,6\%) tienen unos ingresos entre I.200 y I.800 euros y un porcentaje similar (el 26,4\%) entre I. 800 y 3.000 euros. Para el conjunto de la población navarra la renta media por hogar en

Tabla 3. Distribución de los afectados por sexo y edad (absolutos y porcentajes)

\begin{tabular}{|c|c|c|c|c|c|c|}
\hline \multirow{3}{*}{ Grupos de edad } & \multirow{2}{*}{\multicolumn{2}{|c|}{ Total }} & \multicolumn{4}{|c|}{ Sexo } \\
\hline & & & \multicolumn{2}{|c|}{ Mujer } & \multicolumn{2}{|c|}{ Hombre } \\
\hline & $\mathbf{N}$ & $\%$ & $\mathbf{N}$ & $\%$ & $\mathbf{N}$ & $\%$ \\
\hline 25 a 34 & 20 & 14,3 & 16 & 11,4 & 4 & 2,9 \\
\hline 35 a 44 & 43 & 30,7 & 28 & 20,0 & 15 & 10,7 \\
\hline 45 a 54 & 40 & 28,6 & 25 & 17,9 & 15 & 10,7 \\
\hline 55 a 64 & 26 & 18,6 & 22 & 15,7 & 4 & 2,9 \\
\hline 65 y más & 10 & 7,1 & 7 & 5,0 & 3 & 2,1 \\
\hline No contesta & 1 & 0,7 & 1 & 0,7 & 0 & 0,0 \\
\hline Total & 140 & 100,0 & 99 & 70,7 & 41 & 29,3 \\
\hline
\end{tabular}

Fuente: Elaboración propia a partir de los datos del trabajo de campo. 
2010 era de 2.867 euros (INE, 20I3). De hecho un $52, \mathrm{I} \%$ declara no tener dificultades para "llegar a fin de mes" en tanto que en el conjunto de los hogares navarros ese porcentaje se elevaba al $65,5 \%$ (INE, 20I3).

\subsection{Perfil medico-sanitario}

A ocho de cada diez (un $80,8 \%$ ) les fue diagnosticada la esclerosis múltiple entre los 20 y los 44 años, con solamente un 4,3\% en los que se detectó antes de dicha edad. El diagnóstico en los varones es más precoz, como puede apreciarse en la Figura I.

La mitad de los encuestados (un 49,9\%) llevaba entre 5 y I 5 años diagnosticada, un I3,6\% entre I 6 y 20 años, y en otro I7,I \% de los casos habían pasado más de 20 años desde el diagnóstico. Seis de cada diez afectados (un 6 I,4\%) tenían reconocido algún grado de discapacidad: en el 39,5\% de los casos ésta se situaba entre el $33 \%$ y el $64 \%$, en el $37,2 \%$ era del $65 \%$ de discapacidad o superior y en un $23,3 \%$ era menor del $33 \%$. La diferencia por sexos es significativa: el $4 \mathrm{I}, 7 \%$ de las discapacidades femeninas eran del $65 \%$ o superior, mientras que en el caso de los varones el porcentaje era del $26,9 \%$.

Los síntomas asociados a la esclerosis múltiple más citados por los encuestados -los padecen siempre o a menudo- son la fatiga, en el 72,0\% de los casos, la falta de equilibrio, en un 62,I \%, y síntomas motores, con un 56,5\% (Figura 2). Igualmente casi la mitad de los afectados manifiestan dificultades sensitivas, urinarias o espasticidad muscular. Con porcentajes menores pero también altamente significativos se señalaron disfunciones sexuales, síntomas visuales, dolor, dificultad de control de esfínteres,... Es especialmente significativo la diferencia por sexos en los síntomas de dolor: la

\section{Figura 1. Diagnóstico en función de la edad y sexo. Porcentajes}

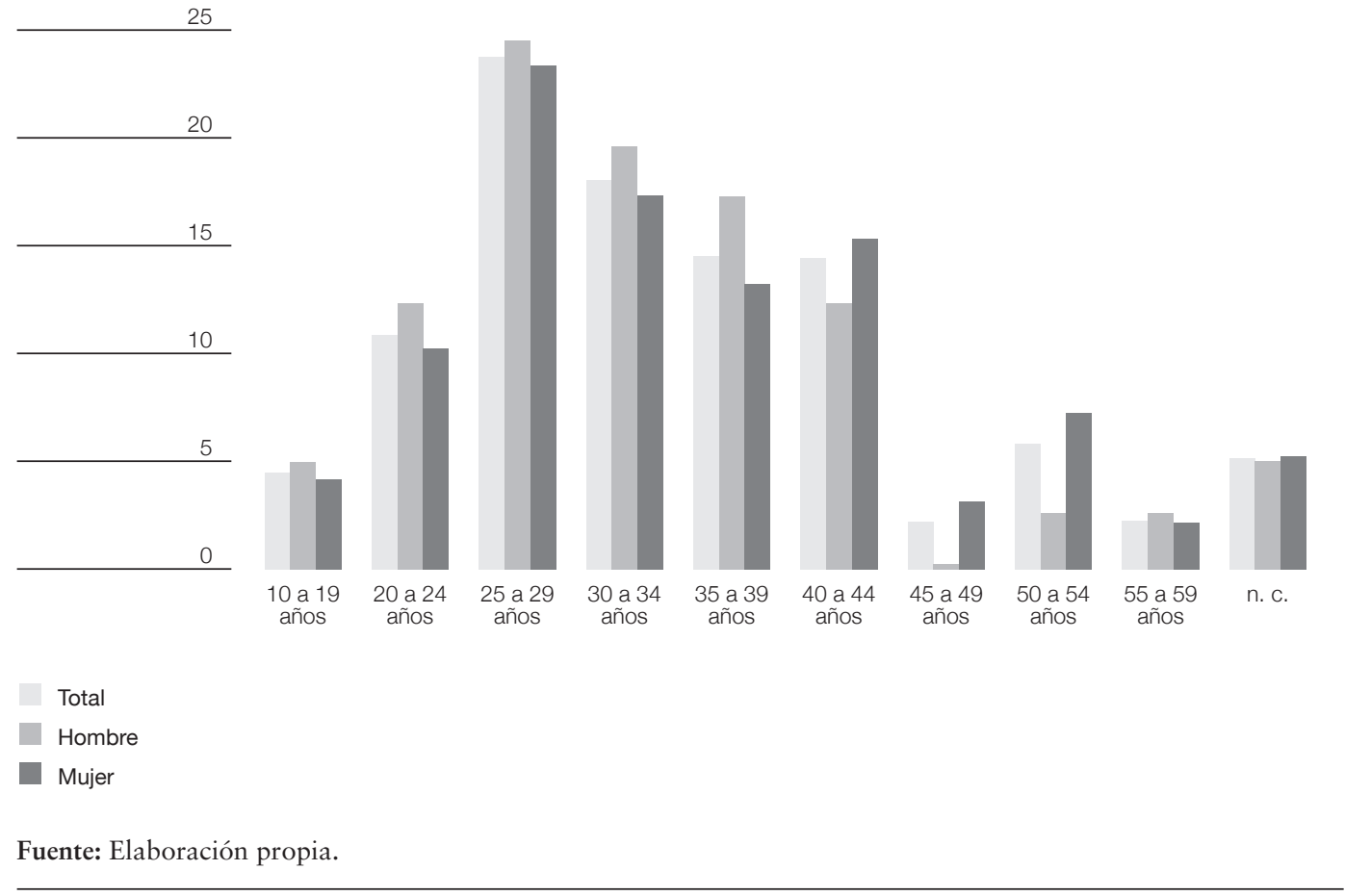


Figura 2. Síntomas asociados a la E.M. más citados por los encuestados (respuesta múltiple)

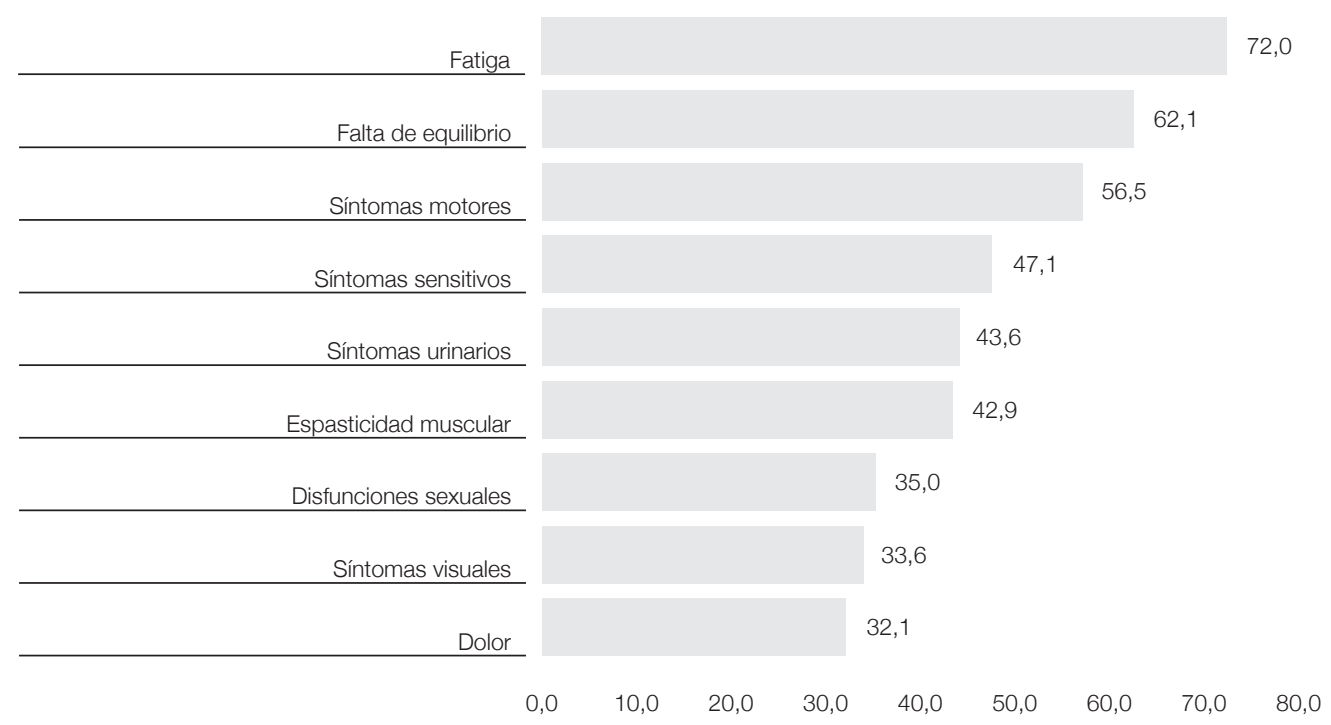

Fuente: Elaboración propia.

población masculina manifiesta sufrirlo siempre o a menudo en un $36,6 \%$ de los casos mientras que en las mujeres el porcentaje es del 20,3\%.

Un I3,6\% de los encuestados afirmaba tener un familiar consanguíneo que también padecía esclerosis múltiple, siendo una especificidad notablemente más presente en la población femenina (un I 6,2 \%) que entre los varones $(7,3 \%$ de casos).

Casi la quinta parte de los afectados (un 19,3\%) manifestaba no tomar ningún tratamiento para la enfermedad (Figura 3), situación sensiblemente más frecuente entre los varones (un $26,8 \%$ no seguía ningún tratamiento farmacológico) que entre las mujeres (así ocurría en un $16,2 \%$ de la población femenina).

Por otro lado, dos tercios de las personas encuestadas siguen algún tratamiento farmacológico en relación con los síntomas asociados. Los más frecuentes están relacionados con la espasticidad (un 30,0\% de los afectados la maneja con algún tipo de fármaco), con la
Figura 3. Evolución del porcentaje de afectados con tratamiento farmacológico para la E.M.

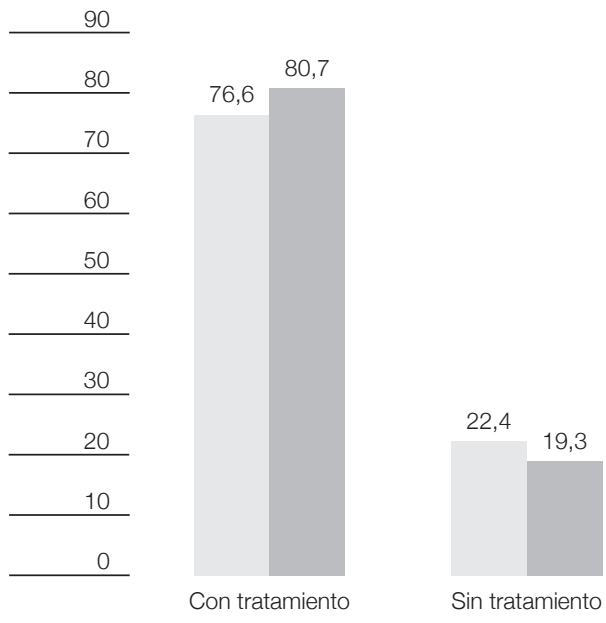

2006

2012

Fuente: Elaboración propia. 
fatiga (un $26,4 \%$ lo hace), con el dolor (un 22, I \%), la depresión (un 22, I \%) o el insomnio (un 2 I,4\%). La diferencia más significativa por sexos se producía en relación con la depresión: un $25,3 \%$ de la población femenina seguía un tratamiento mientras que solamente lo hacían un $\mathrm{I} 4,6 \%$ entre la masculina. Al igual que en los tratamientos específicos para manejar la enfermedad, en relación con los síntomas asociados es superior el porcentaje de varones que no siguen un tratamiento (el 43,9\%) que el encontrado entre las mujeres (un $3 \mathrm{I}, 3 \%$ ).

Los tratamientos alternativos son utilizados por más de un tercio de los afectados (el 35,0\%), siendo los más frecuentes la herboristería (un I 5, O \% afirmaban utilizarlo), los cuidados nutricionales (un $\mathrm{I} 4,3 \%$ ), la homeopatía (un 9,3\%) o la acupuntura (el 7, I \% de los casos).

En el 53,6\% de los casos el entrevistado no sabía si le habían aplicado la escala EDSS de Kurtzke (Kurtzke, I983), considerada una medida de referencia; un I 5,0\% confirmaban que sí la habían pasado aunque solamente el 7 I, $4 \%$ de éstos conocían los resultados. Más de la mitad de los casos (un 53,3\%) habían obtenido valores que oscilaban entre 4,0 y 5 , ○.

\section{3. Áreas de calidad de vida}

Independencia y empoderamiento. $\mathrm{El}$ 43,6\% de los encuestados necesita ayuda de otras personas para realizar alguna de las actividades básicas de la vida diaria (Figura 4), situación más frecuente entre la población femenina (un 49,5\%) que entre la masculina (el 29,3\%).

Entre quienes necesitan ayuda, las necesidades vitales más frecuentes están relacionadas con los desplazamientos fuera del hogar (un 52,5\%), con bañarse (un 39,4\%), con vestirse (un $29,5 \%$ ), o con levantarse/acostarse (un 27,8\%). Por lo que se refiere a otro tipo de necesidades no vitales pero importantes, destacan la ayuda para poder ir de compras (el 63,9\% de quienes necesitaban ayuda), en la limpieza de la casa (un 59,0\%), para la realización de gestiones y papeleo (un $47,5 \%$ ), o para preparar la
Figura 4. Necesidad de ayuda para las actividades de la vida diaria. Porcentajes

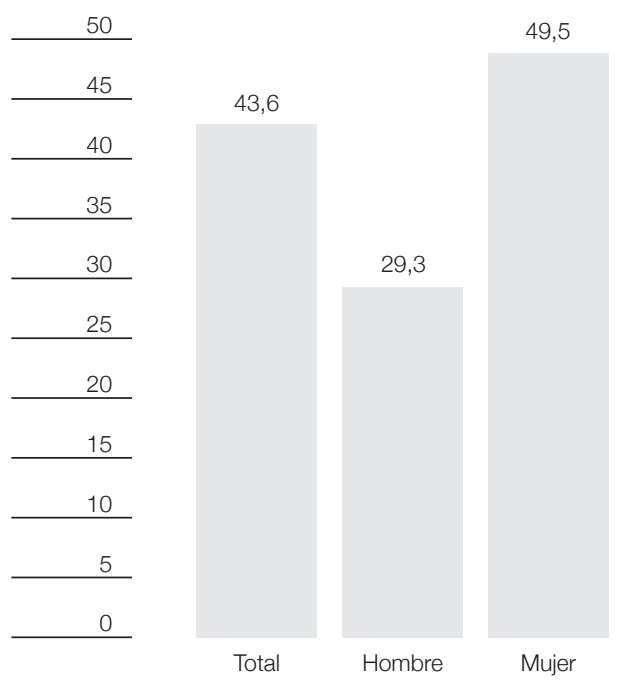

Fuente: Elaboración propia.

comida (un 32,8\%). Un 44,3\% afirman utilizar algún producto de apoyo. Tres de cada diez encuestados (un 30,0\%) utiliza muleta/ bastón, un $17,9 \%$ una silla manual, el 9,3\% una silla a motor, un $5,7 \%$ un andador y un $6,4 \%$ algún otro tipo de producto de apoyo. Casi las dos terceras partes (un 64,5\%) de quienes las utilizan manifiestan hacerlo de forma permanente. Un 30,0\% de los entrevistados declara tener tarjeta de estacionamiento para minusválidos y otro I 2, I \% dice necesitarla aunque no dispone de ella.

Asistencia continua. La gran mayoría (un $85,0 \%$ ) declara que necesita algún tipo de servicio de apoyo, especialmente más reclamado por la población femenina (un $89,9 \%$ frente al $73,2 \%$ entre los varones). Los más demandados son fisioterapia (por un 58,6\% de los encuestados), yoga/pilates/estiramientos (por el $45,0 \%$ ), más información sobre la enfermedad (por un 28,6\%), servicio de ayuda a domicilio (un $23,6 \%$ ) o servicio de viajes y vacaciones con apoyo (un 22, I \%). 
Promoción de salud y prevención de la enfermedad. Un 42, I \% considera que su salud durante los últimos doce meses ha sido buena, mientras que un I7,I \% la considera mala. Entre quienes llevan menos años diagnosticados es más bajo el porcentaje que valora positivamente su salud (un $33,3 \%$ para menos de 5 años, un $44,8 \%$ entre 5 y I 5 años, y un 4I,9 \% para más de I 5 años). Por otro lado, la valoración negativa es notablemente más intensa entre las mujeres (un 2I,2\%) que entre la población masculina (solamente el $7,3 \%$ la considera mala). En una escala de I (muy negativa) a ro (muy positiva) la puntuación media respecto a la calidad de vida de la que disfrutan se situaba en 6,5 puntos. Un $35,0 \%$ han recibido ayuda psicológica en alguna ocasión: un I 6,4 \% la utiliza de manera puntual, un I3,6\% solamente cuando le diagnosticaron la enfermedad y el 5, $0 \%$ de manera continua desde el diagnóstico.

Transporte. Un $86,3 \%$ tiene vehículo propio. De ellos un 6I,5\% manifiesta que no necesita ninguna adaptación y un $28,2 \%$ sí tiene realizadas las adaptaciones necesarias para su manejo. El resto de los casos, aunque las necesitaría, no dispone de ellas. Solamente un $8,5 \%$ de los propietarios de vehículos habían solicitado alguna subvención para llevar a cabo tales adaptaciones.

Vivienda y accesibilidad. El 28,8\% de los encuestados dice que el acceso a su vivienda no ha necesitado ningún tipo de adaptación. Por el contrario, un $47,5 \%$ declara que ya ha sido adaptado en tanto que el 23,8\% manifiesta que la accesibilidad de su hogar no está adaptada a las necesidades derivadas de su enfermedad. En lo relacionado con la adaptación de la vivienda en sí, un $42,4 \%$ ya ha realizado las adaptaciones necesarias, mientras que otro I $2,9 \%$ expresa que sería necesario realizar dichas adaptaciones. La falta de adaptación tanto de los accesos como de la propia vivienda está relacionada, en primer lugar, con problemas económicos y, en segundo lugar, con problemas técnicos de la vivienda.

Empleo. El 30,7\% de los entrevistados estaba trabajando activamente, un $46,4 \%$ tenía reconocida una incapacidad laboral, un I I, $4 \%$ se encontraba en situación de desempleo, y el resto en otras situaciones -tareas del hogar, estudiando, etc.-. La actividad laboral se reduce notablemente a medida que aumenta la edad -el $55,0 \%$ en el tramo de 25 a 34 años y solamente el 7,7\% en el de 55 a 64 años-. El 22, I \% de los encuestados tuvieron problemas en su trabajo al conocerse su diagnóstico, siendo lo más frecuente el despido (en un 4I,9\% de los casos), la incomprensión y pérdida de confianza (en el 22,6\%) o la presión para dejar el trabajo (Figura 5).

Los ingresos de la unidad familiar proceden en un I 8,6\% exclusivamente de la persona afectada, mientras que en otro $62,9 \%$ de los

Figura 5. Principales dificultades en el trabajo entre quienes afirman que las tuvieron. Porcentajes

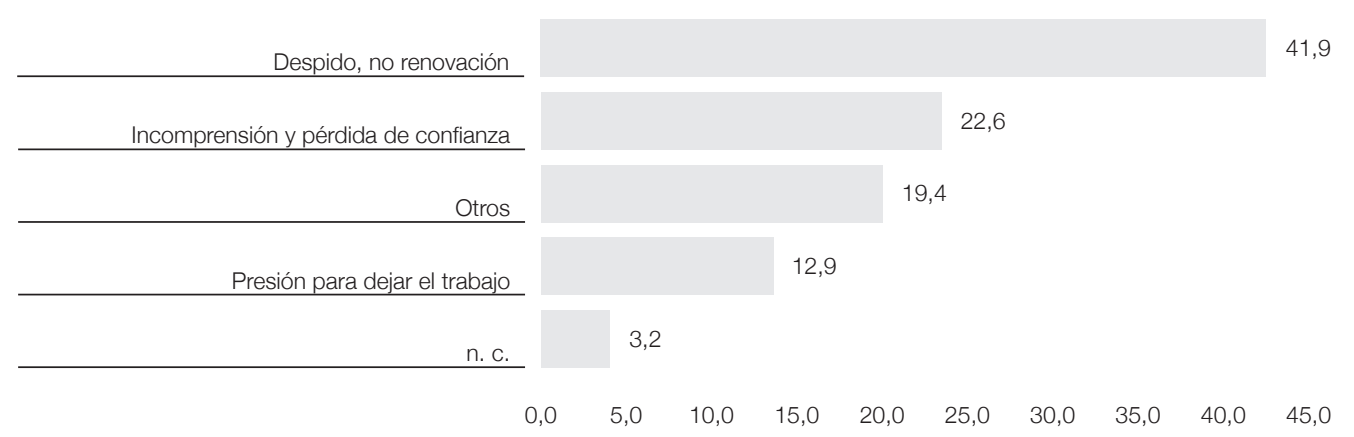

Fuente: Elaboración propia. 
casos los ingresos del afectado son una parte más junto con la de otros miembros del hogar.

Pensiones y ayudas. Un I $8,6 \%$ de las personas entrevistadas tenían realizada una valoración de su dependencia de acuerdo con la normativa vigente, y otro 2,I \% estaba a la espera de obtenerla. Esta valoración era notablemente más frecuente a medida que avanzaba la edad: era de un $5,0 \%$ en el tramo de edad de 25 a 34 años y llegaba hasta el 30,0\% entre los encuestados de 65 años y más. De las valoraciones realizadas un $57,7 \%$ pertenecen al primer grado o "dependencia moderada" (38,5\% para el nivel I y I9,2 \% para el nivel II), un 26,9\% para el segundo grado o "dependencia severa" ( I 9,2 \% y $7,7 \%$ respectivamente) y un $\mathrm{I} 5,3 \%$ para el tercer grado o "gran dependencia" ( I I, 5 \% y $3,8 \%$ según nivel I y II). Solamente un I3,6\% del total de entrevistados afirman recibir algún servicio o prestación económica por su dependencia. Las principales prestaciones son de ayuda económica para asistencia personal (un $52,6 \%$ de las mismas), y teleasistencia (el 26,3\%).

\section{Discusión}

La evolución en el paradigma desde el que se contempla la discapacidad en general y, en concreto, la esclerosis múltiple, ha estado marcada por los cambios en el concepto de salud, en el avance en los derechos sociales subjetivos y en la implantación progresiva de un modelo inclusivo para su abordaje. Todo ello, como hemos podido constatar en la investigación, ha llevado a cimentar un constructo en el que los aspectos médicos y asistenciales son una parte significativa, pero solo una parte de un abordaje integral. En todo caso la puesta en cuestión en el momento actual en toda Europa, y en España en particular, de las políticas de Bienestar, deja en una situación de incertidumbre su dirección futura.

La mayor parte de las investigaciones realizadas en España han sido a nivel local, con una horquilla muy dispersa en cuanto a la prevalencia de la enfermedad. A nivel estatal, y de acuerdo con la revisión efectuada, estimamos que las cifras que circulaban hasta ahora están notablemente infravaloradas. Partiendo tanto de los registros primarios de Navarra como de la encuesta EDAD 2008, y contrastando sus resultados, puede afirmarse que la prevalencia es, al menos, de 78 casos por I00.000 habitantes para la Comunidad Foral de Navarra y que alcanza los I 44 casos en el conjunto de España -entre la población de 6 o más años-.

Desde un punto de vista metodológico se ha coincidido con las principales investigaciones de objetivos similares en la limitación del universo de la encuesta a los diagnosticados que eran miembros de las Asociaciones del ámbito de referencia. Aún así, para el caso de Navarra se ha dispuesto de un "N" de partida que alcanzaba casi la mitad de quienes figuraban en los registros oficiales y, por otra parte, el nivel de respuesta se sitúa en la banda alta de los obtenidos en estudios similares.

En la comparación tanto con el anterior estudio de ADEMNA en 2006 como con otros citados, el perfil sociodemográfico de las personas con esclerosis múltiple permanece bastante estable. Una media de edad ligeramente inferior a los 50 años, en torno a dos tercios de mujeres, menos de uno de cada diez vive solo, la tercera parte recibe una pensión de incapacidad y más de la mitad de los hogares no superan unos ingresos de $\mathrm{r} .800$ euros mensuales. Sin embargo, las diferencias sí son destacables respecto a la población general entre los que, por ejemplo, se duplica el porcentaje de hogares individuales o disponen de una renta media de 2.867 euros mensuales por hogar. Este contraste permite visualizar el importante grado incapacitante de la enfermedad, con un grave impacto laboral y, por ende, con su correlato socioeconómico.

En lo referido al perfil médico-sanitario, se confirma un diagnóstico mayoritario entre los 20 y 44 años y que seis de cada diez tienen reconocido algún tipo de discapacidad -en más de un tercio de los casos igual o superior al $65 \%$-. También se confirman la fatiga, la falta 
de equilibrio y los síntomas motores como los más frecuentes entre los afectados. Sin embargo, aumenta de manera significativa los afectados que siguen un tratamiento farmacológico: solamente dos de cada diez no lo hacen en la actualidad, situación más frecuente entre los varones. Y el recurso a terapias "alternativas" sigue siendo utilizado por más de un tercio de los afectados, especialmente herboristería, cuidados nutricionales, homeopatía o acupuntura.

El enfoque del estudio, fundamentado en el nuevo paradigma de la discapacidad, nos ha permitido detectar que, más allá del estricto espacio clínico, existe toda una serie de dificultades relacionadas con el entorno que inciden directamente en la calidad de vida de los afectados. Se destacan de manera especial algunos ámbitos:

- En relación con su autonomía, casi la mitad de los encuestados necesitan ayuda de otras personas para realizar algunas de las actividades básicas de la vida diaria, especialmente desplazamientos fuera del hogar, bañarse, vestirse o levantarse/ acostarse; y es algo cuyas cifras permanecen estables respecto al estudio de 2006. Aumenta, incluso, la cifra de quienes utilizan algún producto de apoyo -también casi la mitad de los afectados- destacando muletas/bastones y sillas manuales, y las dos terceras partes de los usuarios lo hacen de manera permanente.

- Más allá de las tareas personales, ocho de cada diez afectados necesita utilizar algún tipo de intervención terapéutica o de servicios de apoyo, destacando especialmente los de fisioterapia, si bien la presencia de estos últimos desciende ligeramente respecto a 2006. En cambio está en ascenso la utilización de servicios de yoga/pilates/ estiramientos o la ayuda a domicilio.

- La autopercepción sobre el propio estado de salud es un marcador importante de la vivencia personal de la enfermedad. Si bien cuatro de cada diez consideran que su salud ha sido buena en el último año, hay que destacar que esta frecuencia es directamente proporcional al número de años pasados desde el diagnóstico. El impacto del diagnóstico tiene un importante efecto negativo en esa percepción, que va atemperándose con el tiempo, posiblemente influido por un mayor conocimiento y con habilidades personales para su manejo. De hecho, más de la tercera parte ha recibido ayuda psicológica en alguna ocasión.

- La mitad de los encuestados habían tenido que adaptar el acceso a su vivienda pero otra cuarta parte declara que no lo está, aún necesitándolo. También cuatro de cada diez tuvo que adaptar la propia vivienda y uno de cada diez necesitaría hacerlo y no lo ha hecho, básicamente, por dificultades económicas. Llama poderosamente la atención que esta última necesidad se eleva a más de un tercio de los afectados en el estudio estatal llevado a cabo por FELEM en 2006 , sin que haya evidencias para aventurar si la diferencia se debe a una evolución positiva o una cuestión de ámbito de la investigación.

- Las dificultades de movilidad y la falta de adaptación del transporte público da lugar a que la inmensa mayoría disponga de vehículo propio. Si bien tres de cada diez han adaptado el mismo a sus necesidades, todavía uno de a cada diez no lo ha hecho aunque lo necesitaría y, al igual que en el caso de la vivienda, las razones son fundamentalmente económicas.

- Tres de cada diez estaban laboralmente ocupados y uno de cada diez se encontraba desempleado, en tanto que casi la mitad de los encuestados tenía reconocida una incapacidad laboral. Lamentablemente, una cifra que se mantiene estable respecto al estudio de 2006, es que más de la quinta parte de los encuestados tuvieron problemas en su lugar de trabajo al conocerse el diagnóstico de su enfermedad.

- A pesar de las carencias del sistema de valoración de la dependencia para 
comprender la especificidad de la esclerosis múltiple, dos de cada diez afectados tienen realizada una valoración. De ellas, la cuarta parte se reconoce como una dependencia severa (grado 2 ) y un I $5,3 \%$ como gran dependencia (grado 3 ). La falta de adaptación a las necesidades de la enfermedad también se ponen en evidencia en el tipo de ayudas prestadas: más de la mitad es una ayuda económica y una cuarta parte en forma de teleasistencia.

En conclusión, estamos ante una enfermedad en la que hoy por hoy no hay una respuesta curativa, con opciones terapéuticas paliativas que alivian su cronicidad, y en la que los aspectos que inciden en la mejora de la calidad de vida son fundamentales. Y en ese reto, no basta con intervenir en el entorno individual sino que las actuaciones en el entorno resultan esenciales para avanzar.

Los resultados de la investigación llevada a cabo nos muestran mejoras significativas en aspectos médico-sanitarios y algunas otras áreas, pero también déficits importantes sobre todo en lo relacionado con la autonomía y movilidad de los afectados, y en su ámbito laboral y relacional. Resulta necesario asimismo aumentar la "visibilidad" de la esclerosis múltiple de cara especialmente a la valoración de su dependencia y otras necesidades, dificultada por su carácter intermitente. En una palabra, posibilitar su integración activa y plena en la sociedad.

Sería interesante que futuros estudios sobre la situación y necesidades de las personas afectadas por esclerosis múltiple, si no resultase factible su carácter estatal, se llevasen a cabo en sus respectivos ámbitos con metodologías que permitan su comparabilidad. Una mejora significativa sería la inclusión en el universo de estudio de afectados que se encuentren fuera de los ámbitos asociativos. Asimismo, y como futuras líneas de investigación, proponemos la incorporación del análisis de los determinantes sociales de la salud que pueden afectar a las personas con diagnóstico de esclerosis múltiple y, como estrategia metodológica, la incorporación del análisis biográfico, a través de técnicas de historia de vida, para poder analizar las etapas clave en la vida de una persona afectada. 


\section{Referencias bibliográficas}

ADEMNA (2006): Esclerosis múltiple en Navarra: realidad, necesidades sociales y calidad de vida. Pamplona: Asociación ADEMNA.

Banwell, B. et al. (2013): "Editors' welcome and a working definition for a multiple sclerosis cure". Mult Scler Relat Disord (Netherlands), 2 (2): $65-67$.

Compston, A. y Coles, A. (2008): "Multiple sclerosis”. Lancet, 372 (9648): I 502-I 5 I 7.

Correale, J. et al. (2002): "Enfermedades desmielinizantes”, en Micheli, F. et al. (dirs.): Tratado de Neurología Clínica. Buenos Aires: Editorial Médica Panamericana.

Delgado-Mendilívar, J.M. et al. (2005): "Estudio de la calidad de vida en la esclerosis múltiple". Rev Neurol, 4I: 257-262.

Duquette, P. et al. (I992): “The increased susceptibility of women to multiple sclerosis". Can J Neurol Sci, I9: 466-47I.

EDE Fundazioa (2003): Las personas afectadas de esclerosis múltiple y sus necesidades: Estudio de los y las asociadas a la Fundación Esclerosis Múltiple Eugenia Epalza Fundazioa. Bilbao: EDE Fundazioa.

España. Ley 39/2006, de I4 de diciembre, de Promoción de la Autonomía Personal y Atención a las personas en situación de Dependencia, Boletín Oficial del Estado, núm. 299 de 15 de diciembre de 2006, p.44I42-44I 56 (en línea). <http://www.boe.es/ boe/dias/2006/I 2/I 5/pdfs/A44I 42-44I 56.pdf>, acceso I 8 de noviembre de 2012.

European Multiple Sclerosis Platform (2008): Code of good practices in multiple sclerosis. Brussels: EMSP.

FEDEMA (2008): Atención social, estudio de necesidades y costo socio-laboral de la esclerosis múltiple y otras enfermedades similares. Sevilla: Federación FEDEMA.

FELEM (2007): Esclerosis múltiple en España: realidad, necesidades sociales y calidad de vida (en línea). <http://issuu.com/esclerosismultipleespana/
docs/memoria_invest_calidad_de_vida/I>, acceso I 2 de noviembre de 2013 .

Giovannoni, G. (20I3): "Ectrims 20I2: Treatment Highlights”. Mult Scler Relat Disord, 2 (2): 68-72.

Gobierno de España (20II): Estrategia Española sobre Discapacidad 2012-2020. Ministerio de Sanidad, Política Social e Igualdad, Madrid: Real Patronato sobre Discapacidad.

Gobierno de Navarra (2010): Registro de casos de esclerosis múltiple. Departamento de Salud. Pamplona: Gobierno de Navarra.

Huete, A. y Díaz, E. (2009): Estudio sobre situación de necesidades sociosanitarias de las personas con enfermedades raras en España. Madrid: Obra Social Caja Madrid.

Instituto Nacional de Estadística (20I I): Encuesta de Presupuestos Familiares 20 I I (en línea). <http://www.ine.es/jaxi/ menu.do?type $=$ pcaxis $\&$ path $=\%{ }_{2} \mathrm{Ft}_{25} /$ p458\&file=inebase $\& \mathrm{~L}=0>$, acceso ro de enero 2013 .

- (2010): Encuesta de Condiciones de Vida 2010 (en línea). <http://www.ine.es/jaxi/ menu.do? type $=$ pcaxis $\&$ file $=$ pcaxis $\&$ path $=\% 2$ Ft25\% 2Fp453\%2F\%2Fa2Oro>, acceso ro de enero de $20 \mathrm{I} 3$.

- (2008): Encuesta sobre Discapacidades, Autonomía personal y situaciones de Dependencia: EDAD 2008 (en línea). <http://www.ine.es/jaxi/menu. do? $\mathrm{L}=o \&$ type $=$ pcaxis\&path $=/ \mathrm{tI} 5 /$ p4I8\&file=inebase $>$, acceso ro de enero de 2013.

Kurtzke, J.F. (I997): “The epidemiology of multiple sclerosis", en Raine, CS. et al. (eds.): Multiple sclerosis clinical and pathogenetic basis. London: Chapman \& Hall Medical.

- (I993): "Epidemiologic evidence for multiple sclerosis as an infection". Clin Microbiol Rev, $6(4): 382-427$.

- (1983): "Rating neurologic impairment in multiple sclerosis: an expanded disability status 
scale (EDSS)". Neurology (Cleveland),

33: I444-I 452.

Mallada, J. (I999): “Epidemiología de la esclerosis múltiple en España: datos de prevalencia e incidencia”. Rev Neurol, 29 (9): 864-867.

Multiple Sclerosis International Federation (2002): Principios para promover la calidad de vida de las personas con esclerosis múltiple. Madrid: MSIF.

Navarra. Ley Foral I 5/2006, de I4 de diciembre, de Servicios Sociales, Boletín Oficial de Navarra, núm. I 52, de 20 de diciembre de 2006 (en línea). <http://www.lexnavarra. navarra.es/detalle.asp? $\mathrm{r}=\operatorname{IO} 855>$, acceso I 8 de noviembre de $20 \mathrm{I} 2$.

Organización Mundial de la Salud (2009): Documentos básicos: Constitución de la OMS (4 $47^{\mathrm{a}}$ ed.), Ginebra: OMS.

- (I978): Atención primaria de salud: Informe de la Conferencia Internacional sobre Atención Primaria de Salud. Alma-Ata (URSS) 1978. Ginebra: OMS.

Pérez, L.C. (2006): Discapacidad y asistencia sanitaria. Madrid: CERMI.
Pineault, R. y Potvin, L. (2003): “Milton Terris's career”. J Public Health Policy, 24: 77-8I.

Ponte, C. (2008): Conceptos fundamentales de la salud a través de su historia reciente (en línea). <http://8 5.I 52.37.8/recursos/2008/ SaludyCiudadania.pdf $>$, acceso $\mathrm{I} 5 \mathrm{de}$ noviembre de 20 I I.

Raus, J. (I998): “Multiple sclerosis”, en Delves, PJ. y Roitt, IM. (eds.): Encyclopedia of Immunology, ( $2^{\mathrm{a}} \mathrm{ed}$.). London: Academic Press.

Simone, I. et al. (2000): "Early onset multiple sclerosis”. Neurol Sci 21: 86I-863.

Sociedad Española de Neurología (20I3): Día Mundial de la Esclerosis Múltiple (en línea). $<$ http://www.sen.es/saladeprensa/pdf/Linkı 2 . pdf $>$, acceso 23 de noviembre de 2013 .

WHOQOL (I995): “The World Health Organization Quality of Life AssessmentWHOQOL-: position paper from the World Health Organization”. Soc Sci Med, 4I ( IO): I 403-I409. 